\title{
Low Complexity Millimeter Wave Point-to-point Communication: Interference Assessment of BPSK vs QPSK Decomposition
}

\author{
D. Borges ${ }^{1,2}$, P. Viegas ${ }^{2}$, P. Montezuma ${ }^{1,2}$, R. Dinis ${ }^{1,2}$, and M. M. Silva ${ }^{1,3}$ \\ ${ }^{1}$ DEE, FCT Universidade Nova de Lisboa, Portugal \\ ${ }^{2}$ IT, Instituto de Telecomunicações, Av. Rovisco Pais, Lisboa, Portugal \\ ${ }^{3}$ Universidade Autónoma de Lisboa, Portugal
}

\begin{abstract}
Power amplification and spectral efficiencies could be optimized with transmitter structures employing several branches, each one associated to a signal component of the symbol to be transmitted, with an amplifier directly connected to an antenna or an antenna array. Under these conditions the signal components transmitted by the several branches are associated to different beams to increase the separation among data streams from different users. However, even in these conditions interference among users can occur due to superposition of radiation beams carrying signal components from different users. Since the physical security level assured by these structures is not negatively affected, it is crucial the assessment of interference effects in such systems. Under certain limits in misalignments, simulation results show that beam interference has low impact on system performance.
\end{abstract}

\section{INTRODUCTION}

Massive Multi-Input Multi-Output systems (mMIMO), with hundreds or thousands of communicating antennas, are the most prevalent candidates for next generation of wireless communications [1]. Among mMIMO advantages we can name the reduction on the transmitted power and the massive deployment of spatial multiplexing schemes for more efficient bandwidth use $[2,3]$. Thus, the tremendous data rates need for downlink in next generation of wireless systems could be attainable by mMIMO implementations at the base station's (BS) side. Additionally, the use of mmWave bands in $5 \mathrm{G}$ allows to increase the number of antennas in mobile devices, due to the conceivable reduction of antenna dimensions.

The freedom degrees of such mMIMO schemes will alow deployments of layered transmitter and receiver structures where both spectral spectral and amplification efficiencies could be optimized. In such structures, the first layer consists in decomposing the high order constellation symbol to be transmitted into several components with lower peak-to-average power ratio (PAPR). These components, such as bi-phase shift keying (BPSK) or as quadrature PSK (QPSK), are amplified and sent through the channel in parallel $[4,5]$. The second layer is formed by the antenna arrays connected to each amplification branch. The third layer composed by several sets of first and second layers in parallel that perform spatial multiplexing [6]. Figure 1 illustrates the described layered structure.

Improvments in energy efficiency of power amplification are achievable since the original constellation with high PAPR is decomposed into components with lower PAPR that are amplified by nonlinear (NL) amplifiers in each branch. Thus, we may say that first layer minimizes problems that may result from nonlinear distortion effects when high order constellations are employed in transmission. The output of each branch of layer 1 is a connected to an antenna array of layer 2 which transmits the component from the respective branch. The higher order constellation symbol is obtained at channel level through the sum of all the beams of the transmitted components.

Since the bit streams in different branches are uncorrelated, the overall radiation pattern of the antennas connected to the first layer remains omnidirectional, being the radiation pattern only created by the antennas of layer 2 . Nonetheless, it should be mentioned that a information directivity is introduced in the transmitted information since the components associated to the different beams suffer different rotation phases according the azimuthal direction. Previous works already shown that these transmitters have same robustness against interference than transmitters with common beamforming (based on a 2-D array) without penalties on system's performance [9]. However, no assessment was done about the impact of interference among users due to beam misalignments at BS side. Therefore, it seems critical to study the interference effect due to misalignments between the several beams carrying the signal components, which is the purpose of 
this work. This analysis will identify the requirements in alignment precision of the different beams and the applicability of this multi layered structure with layers 1 and 2 , under these impairments.

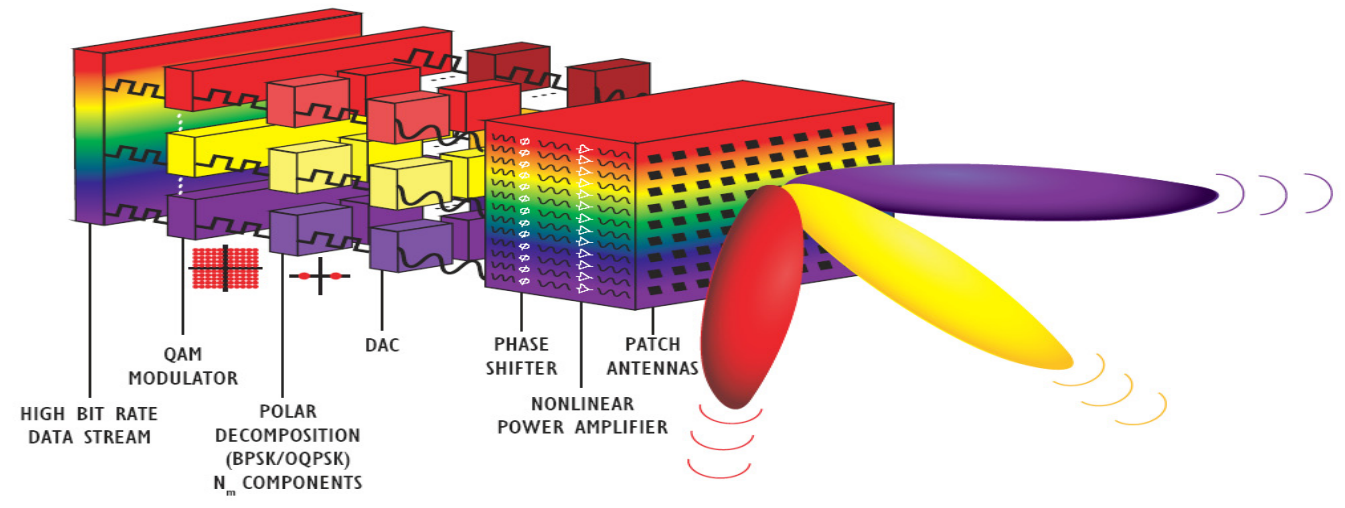

Figure 1. Layered structure with layers 1, 2 and 3.

This rest of this paper is organized as follows: Section 2 characterizes the layered transmitter structure. The characterization of the overall transmission system is done in Section 3 . The assessment of the impact of the mentioned impairments is analyzed in Section 4. Finally, Section 5 summarizes the paper.

\section{MULTI LAYERED TRANSMITTER ARCHITECTURE}

Figure 2 illustrates a mMIMO deployment where users have a layered structure with $N_{m} \times N_{b}$ antenna elements, arranged in $N_{m}$ sets of $N_{b}$ antennas. In each user, beamforming is achieved by a layer 2 with arrays of $N_{b}$ antenna elements connected to each one of the $N_{m}$ amplification branches. At BS side, spatial multiplexing could be implemented by layer 3 with $N_{v} \times T$ antennas, where $T$ antennas are associated to layers 1 and 2 (related to the beams of signal components) and the $N_{v}$ sets of $T$ antennas are used to transmit or receive simultaneously $N_{v}$ different constellation symbols.

To reduce the PARP and maximize efficiency of power amplification layer 1 takes advantage from the fact that multilevel constellations can be decomposed in polar components with the constellation symbols expressed as a function of the corresponding bits $[4,10]$. Being $\mathcal{S}=\left\{s_{0}, s_{1}, \ldots, s(N-1)\right\}$, a constellation with $M$ points (i.e., $\# \mathcal{S}=M$ ), where $s_{n} \in \mathbb{C}$, it is possible associate to each constellation point $s_{n}$ a set of $\mu=\log _{2}(M)$ bits in polar format $\mathcal{B}=\left\{b_{n}^{0}, b_{n}^{1}, \ldots, b_{n}^{(\mu-1)}\right\}\left(\right.$ i.e., $b_{n}^{(i)}=$ $\pm 1=2 \beta_{n}^{(i)}-1, \beta_{n}^{(i)}=0$ or 1$)$. Since there are $M$ constellation points in $\mathcal{S}$ and $M$ different subsets of $\mathcal{B}, \mathcal{B}_{0}, \mathcal{B}_{1}, \ldots, \mathcal{B}_{M-1}$, we may write

$$
s_{n}=\sum_{m=0}^{M-1} g_{m} \prod_{b_{n}^{(i)} \in \mathcal{B}_{m}} b_{n}^{(i)}, n=0,1, \ldots, M-1
$$

which corresponds to a system of $M$ equations (one for each $s_{n}$ and $M$ unknown variables $g_{m}$ ). As stated before, each polar component can be modulated as a BPSK or QPSK with low envelope fluctuations and lower PARP than the original constellation. Each one of these signals components will be amplified separately in each transmission branch. Under these conditions it becomes obvious that power efficiency comes improved due the lower PAPR of the component signals and the possible use of NL amplifiers $[4,5]$. Since the bit streams associated with each branch are uncorrelated the corresponding symbols are as well uncorrelated, whereby the radiation pattern associated to the first layer with $N_{m}$ antennas remains omnidirectional. Despite the omnidirectional radiation pattern, phase rotations of signal components associated to the $N_{m}$ sets of $N_{b}$ antennas change the shape of the resulting signal, which means that constellation points of the transmitted signal maintain their positions at the desired direction $\Theta$ but suffer distortion in other directions. 


\section{SYSTEM CHARACTERIZATION}

The transmitter's configuration has the layered structure shown in Figure 2, were Layer 2 is composed by sets $N_{b}$ antennas each one connected to each amplification branch (for a decomposition of 16-quadrature amplitude modulation (16-QAM) into QPSK or BPSK components we have $N_{m}=2$ and $N_{m}=4$ respectively). Under these conditions each user has a second layer with $T=N_{b} \times N_{m}$ antennas that transmits simultaneously $N_{m}$ symbol components (more exactly the QPSK or BPSK components from 16-QAM constellation) with the radiation beams perfectly aligned.

With the advent of millimeter waves, it is expected an increase in the the number of antennas in the mobile terminals. Despite the massive use of antennas, the higher bit rates are only possible with resort to broadband channels characterized by a frequency selective channel. To cope with channel's frequency selectivity it can be adopted a SC-FDE block transmission technique with a iterative block decision feedback equalization (IB-DFE) receiver whose structure is depicted in Figure $3[7,8,11,12]$. However, it still seems unrealistic to expect more than ten antennas at mobile terminals, which leads us to assume that layer 2 is implemented by arrays with 3 antennas. For a layer 1 based on BPSK components this leads to a number of $T=12$ antennas for each mobile user (for a layer 1 with $N_{m}=2$ branches, i.e., with QPSK components we have $T=6$ ).

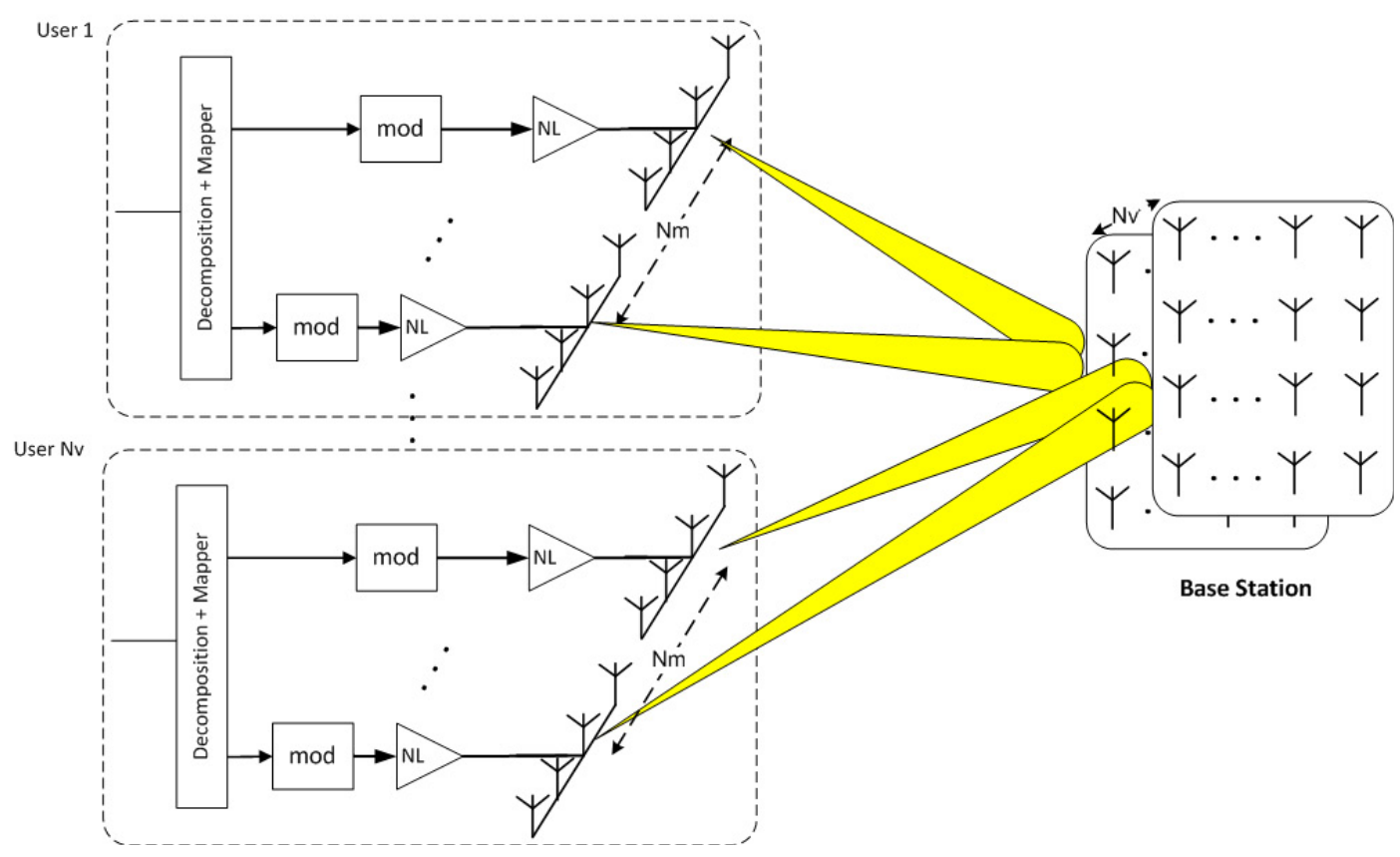

Figure 2. Layered structure with layers 1, 2 and 3.

The mMIMO scenario can be modeled as shown in Figure 1, where exists a point-to-point communication link between $N_{v}$ users, each one with $T=N_{b} \times N_{m}$ antennas and the BS with $R \geq N_{v} \times T$ antennas.

Coupling effects among antennas are avoided by a horizontal spacing of $\lambda$ between antennas of layer 1 and a vertical spacing of $\lambda$ between antennas of layer 2 (it is important to mention that the radiation pattern is still omnidirectional in vertical plane). Interference arises when beams form different user overlap. An obvious consequence is the distortion of the signal resulting due to reinforcement or attenuation of the signal component affect by interference. Directional interferences among users, are due beams misalignments or due to proximity (in the later the distance between users leads to an insufficient angular separation to assure that interfering signals arrive in the direction of the nulls of radiation pattern of the receive array). For comparison purposes, it will be also considered the scenario without interference.

At each user the $t^{t h}$ arrays sends the block of $N$ data symbols $\left\{x_{n}^{(t)} ; n=0,1, \ldots, N-1\right\}$ being $\left\{y_{n}^{(r)} ; k=0,1, \ldots, N-1\right\}$ the received block at the $r$ th receiver's antenna. As usual a cyclic prefix with a length higher than the overall channel impulse response is appended to each transmitted block and removed at the receiver. Thus the corresponding frequency-domain received 
block $\left\{Y_{k}^{(r)} ; k=0,1, \ldots, N-1\right\}$ is given by

$$
\mathbf{Y}_{k}=\left[\begin{array}{lll}
Y_{k}^{(1)} & \ldots & Y_{k}^{(R)}
\end{array}\right]^{T}=\mathbf{H}_{k} \mathbf{X}_{k}+\mathbf{N}_{k}
$$

where $\mathbf{H}_{k}$ denotes the $R \times T$ channel matrix for the $k$ th frequency, with $(r, t)$ th element $H_{k}^{(r, t)}$, $\mathbf{X}_{k}=\left[X_{k}^{(1)} \ldots X_{k}^{(T)}\right]^{T}$ and $\mathbf{N}_{k}$ denotes the channel noise.

For an iterative minimum mean squared error (MMSE) receiver the data symbols for a given iteration can be obtained from the inverse discrete Fourier transform (IDFT) of the block $\left\{\tilde{X}_{k}^{(t)} ; k=\right.$ $0,1, \ldots, N-1\}$, where

$$
\tilde{\mathbf{X}}_{k}=\left[\tilde{X}_{k}^{1} \ldots \tilde{X}_{k}^{(R)}\right]^{T}=\mathbf{F}_{k} \mathbf{Y}_{k}-\mathbf{B}_{k} \overline{\mathbf{X}}_{k}
$$

(more details can be seen in [14]), where $\mathbf{I}$ is an identity matrix and $\alpha=E\left[\left|N_{k}^{(r)}\right|^{2}\right] / E\left[\left|X_{k}^{(t)}\right|^{2}\right]$ is assumed identical for all antennas $t$ and $r, F_{k}$ is the feedforward coefficient, $B_{k}$ is the backward coefficient and $\rho$ denotes the the correlation coefficient that can be computed as described in $[10,12-$ 14]. Interference cancelation is done using $\overline{\mathbf{X}}_{k}=\left[\bar{X}_{0} \ldots \bar{X}_{N-1}\right]$, with $\bar{X}_{k}$ denoting the frequencydomain average values conditioned to the FDE output for the previous iteration. It should be mentioned that in first iteration this receiver is equivalent to a linear frequency-domain MMSE receiver since no information is available about the transmitted symbols and $\overline{\mathbf{X}}_{k}=\mathbf{0}$. Subsequent iterations employ the average values conditioned to the receiver output from previous iteration to remove the residual intersymbol interference (ISI) and inter user interference.

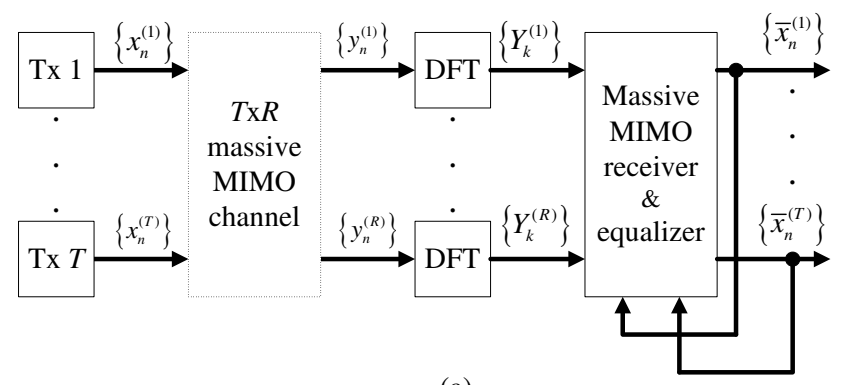

(a)

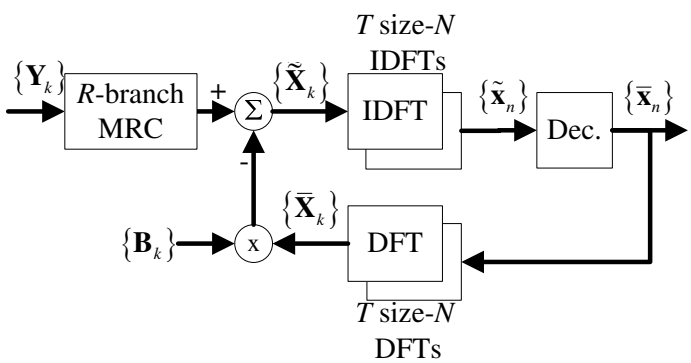

(b)

Figure 3. Overall massive MIMO system for SC-FDE schemes (a) and detail of the massive MIMO receiver and equalization (b).

The high number of antennas involved in communication links aggravates the IB-DFE's computational complexity problem due to the size of channel matrix that must be inverted. To reduce complexity, two low-complexity iterative frequency-domain receivers, denoted as maximum ratio detection (MRD) and equal gain detection (EGD) are also considered as in [15]. Also, having in mind previous results regarding the influence on performance the ratios $R / T$ between receiving and transmitting antennas from [15], it is assumed $R / T>4$ more specifically $R / T=10$.

As mentioned in [15], the MRD receiver is characterized by

$$
\tilde{\mathbf{X}}_{k}=\mathbf{\Psi} \mathbf{H}_{k}^{H} \mathbf{Y}_{k}-\mathbf{B}_{k} \overline{\mathbf{X}}_{k},
$$

and

$$
\mathbf{B}_{k}=\mathbf{\Psi} \mathbf{H}_{k}^{H} \mathbf{H}_{k}-\mathbf{I}
$$


where $\boldsymbol{\Psi}$ denotes a diagonal matrix whose $(t, t)$ th element is given by $\left(\sum_{k=0}^{N-1} \sum_{r=1}^{R}\left|H_{k}^{(r, t)}\right|^{2}\right)^{-1}$, takes advantage of the fact that $\mathbf{H}_{k}^{H} \mathbf{H}_{k} \approx R \mathbf{I}$ which is accurate when both conditions are met: $R \gg 1$ and small correlation between different channels. The EGD receiver is characterized by

$$
\mathbf{B}_{k}=\mathbf{\Psi} \mathbf{A}_{k}^{H} \mathbf{H}_{k}-\mathbf{I}
$$

which takes advantage from the fact that outside the main diagonal of

$$
\mathbf{A}_{k}^{H} \mathbf{H}_{k}
$$

the matrix elements are much lower than the ones form the main diagonal, where $\left(i, i^{\prime}\right)$ th element of the matrix $\mathbf{A}$ is $[\mathbf{A}]_{i, l}=\exp \left(\operatorname{jarg}\left([\mathbf{A}]_{i, l}\right)\right)$, when $R \gg 1$ and small correlation exists between channels associated to different transmit and receive antennas.

\section{PERFORMANCE ASSESSMENT}

Independent trials of Monte Carlo experiments are used to obtain the average results of Bit error rate (BER) for Rayleigh channel (for each value of BER are considered at least 100 error events). Bit error rate (BER) results are based on Monte Carlo simulations to obtain the average results for a Rayleigh channel (for each value of BER are considered at least 100 error events). Layer 1 may have two different configurations: a first one with $N_{m}=2$ where the 16-QAM symbol is decomposed into QPSK components and a second one where the 16-QAM symbol is decomposed in $N_{m}=4$ BPSK components. For both configurations the transmitted symbols $x_{n}$ are selected with equal probability from a 16-QAM constellation. It is also assumed linear power amplification at the transmitter, perfect channel estimation and perfect synchronization conditions at the receiver. A maximum of 4 iterations are considered at the receiver (previous simulations with iterations up to 10 showed that after 4 iterations the improvements in performance did not justify the increase in receiver's complexity). BER results are expressed as function of $\frac{E_{b}}{N_{0}}$, where $N_{0} / 2$ denotes the noise variance and $E_{b}$ represents the energy of the transmitted bits. In terms of interferent users we defined only the scenarios where there in no interference or other where 3 users are appliyng interference. It is considered that these apply the same symbol decomposition as the main user. It is also assumed that only one beam (component) from the interferent users affects all the components from the main user. The interferent beam can be refered as strong or weak realted to the amplitude of the respective component. The simulated interference levels are $-10 \mathrm{~dB},-5 \mathrm{~dB}$ and $-2 \mathrm{~dB}$.

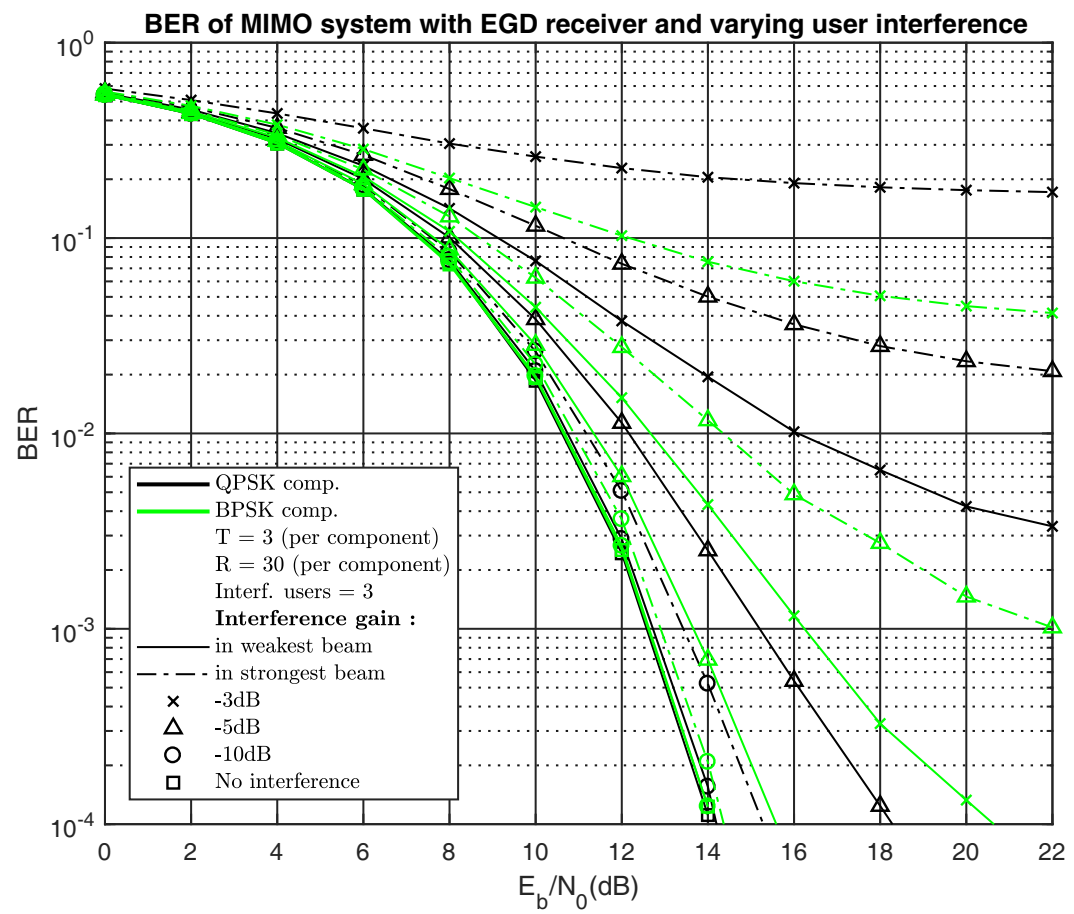

Figure 4. BER performance for Layer 1 and 2 transmitter, EGD receiver and $R / T=10$. 


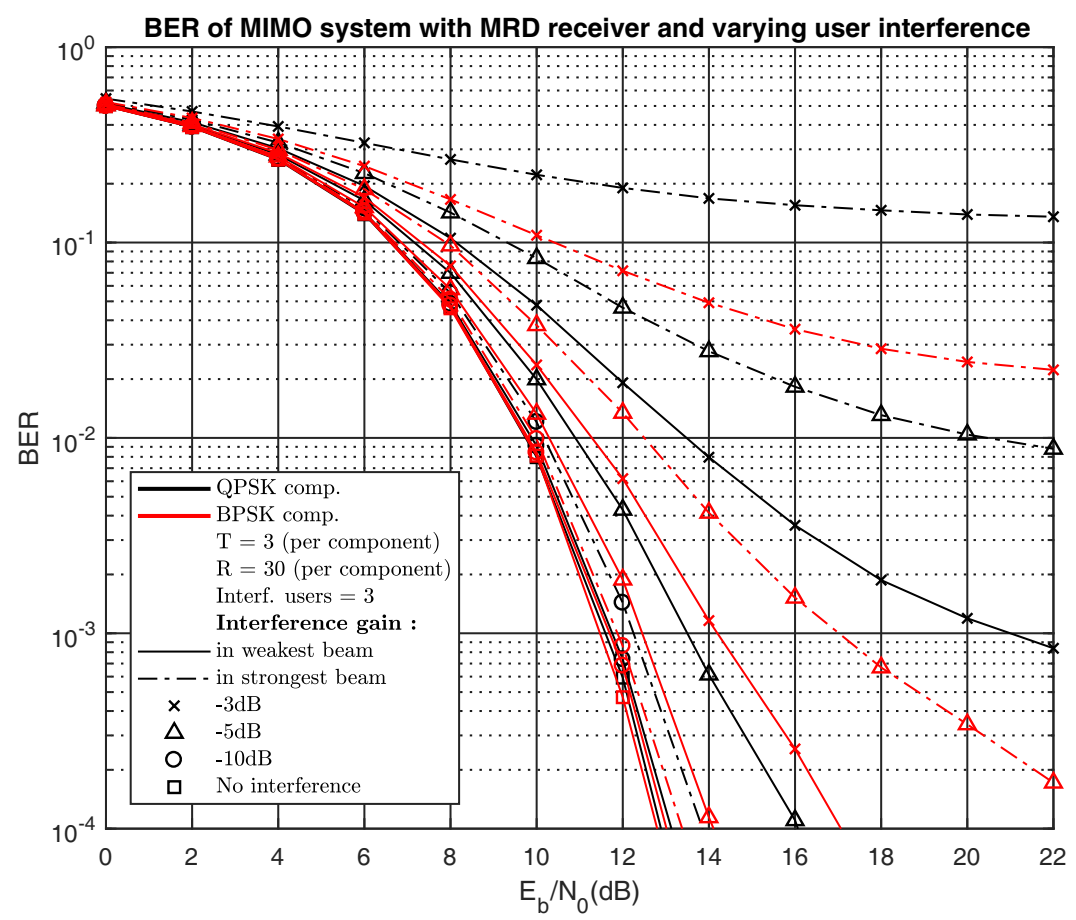

Figure 5. BER performance for Layer 1 and 2 transmitter, MRD receiver and $R / T=10$.

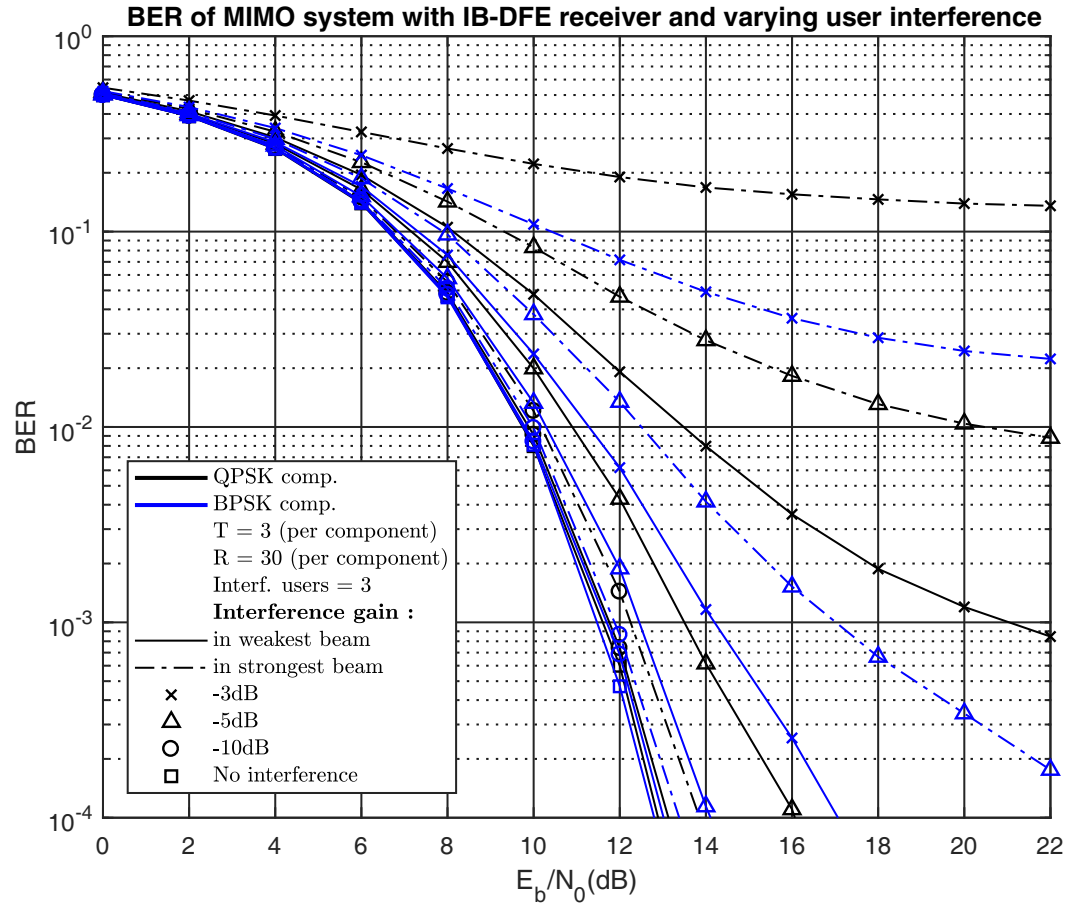

Figure 6. BER performance for Layer 1 and 2 transmitter, IB-DFE receiver and $R / T=10$.

Results shown in Figures 4,5 and 6 depict EGD, MRD and IB-DFE receivers with similar simulation conditions and system configurations based on combination of layer 1 and layer 2 . From the results of Figures 5 and 6 it is also clear that both IB-DFE and MRD receivers have similar performances. As expected, these two receivers perform better than the EGD. Also, the results considering the absense of interference are similar to the ones obtained by a transmitter using only Layer 1 or by a conventional spatial multiplexing scheme, which means that combination of layer 1 and layer 2 does not sacrifice system's performance when interference is nonexistent.

It can be seen that interference with $-10 \mathrm{~dB}$ has very low impact in system performance, inde- 
pendently of the receiver or interferent beam (being the biggest impact around $1 \mathrm{~dB}$ for a BER of $10^{-4}$, for $N_{m}=2$ and interference of the strongest beam). In the situation where there is no interference considered the EGD performance has a degradation of $1 \mathrm{~dB}$ (for a BER of $10^{-4}$ ) compared with the other two receivers.

For all the scenarios where increasing levels of interference are applied, the EGD receiver has an increasing degradation compared to the other two receivers in the same conditions.

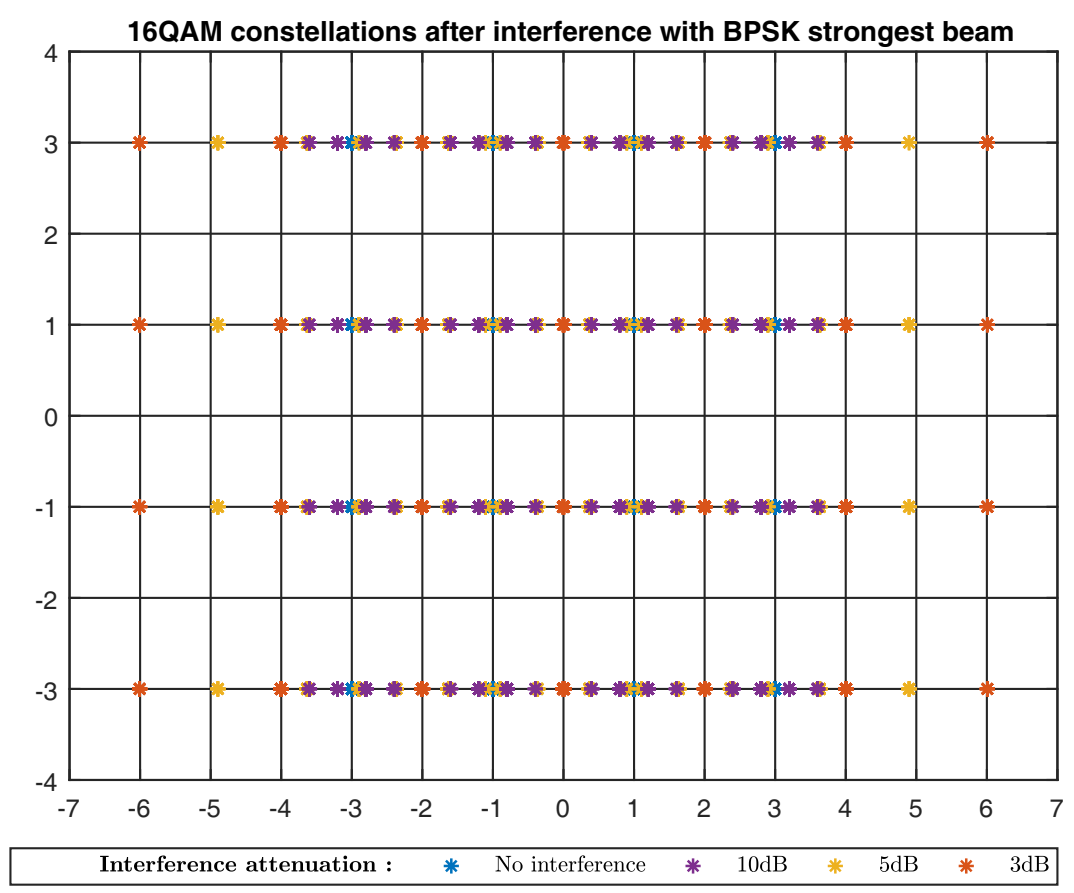

Figure 7. Constellation distortion with interference on the strongest BPSK component.

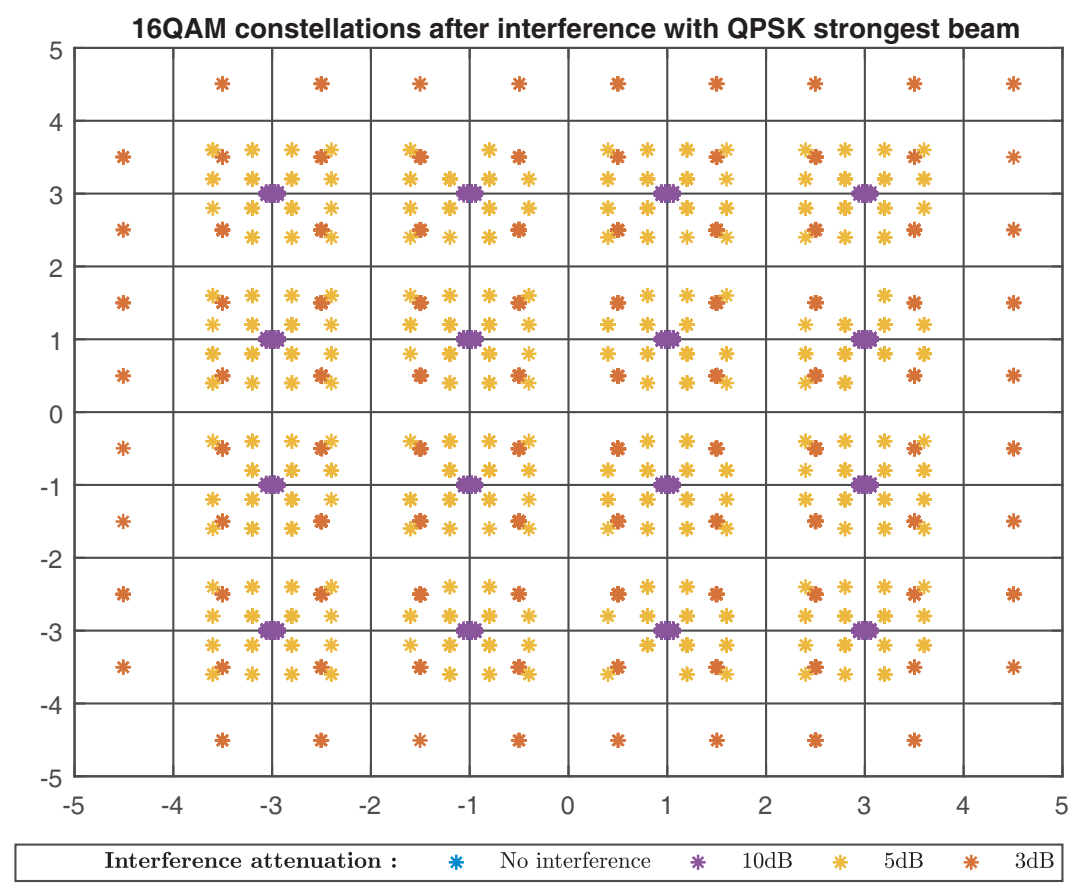

Figure 8. Constellation distortion with interference on the strongest BPSK component.

As an example we can observe the settings of $N_{m}=2$ (QPSK components) with $-3 \mathrm{~dB}$ interference from the weakest beam, where the EGD receiver shows around $4 \mathrm{~dB}$ degradation for a BER of $10^{-4}$, compared to the MRC or IB-DFE receivers. 
Other important result is that the impact in the BER from an interference with a strong beam is much higher than the one coming from a weaker beam, for the same interference levels. This gap is visible by comparing the curves with a solid line and dashed-dot line, representing these two simulated types of interference. This effect gets more noticeable for higher interference gains, independently of the receiver. Furthermore, this impac is more perceptible in the QPSK decomposition than BPSK decomposition. As expected, this behavior is justified by the fact that interferent QPSK components correspond to two BPSK components at same time, and consequently have a stronger distortion effect in the symbols resulting from the combination of the components. The consequence of this interference can be seen in Figures 7 and 8 where it is obvious the higher distortion due to QPSK beam. With interference levels higher than $-3 \mathrm{~dB}$ for weak beam and $-5 \mathrm{~dB}$ for strong beam interference, the receiver's sensitivity compromises the decoding capacity of the system with the studied receivers.

\section{CONCLUSION}

In this paper it was shown that a transmitter based on a double layer structure with information directivity and could be employed even under conditions where interference among users exists. For small interference levels (lower than $10 \mathrm{dBs}$ ) performance results remain practically unaffected by interference. Above this threshold the performances of MRD and IB-DFE receivers are less affected than EGD receiver. However, the effects of interference in this layered system are similar to the ones that affect common beamforming implementations.

\section{ACKNOWLEDGMENT}

This work was supported in part by Foundation for Science and Technology (PhD scholarship SFRH/BD/131093/2017), IT UID/EEA/50008/2013 (plurianual founding and project GLANCES), EnAcoMIMOCo EXPL/EEI-TEL/2408/2013, UID/EEA/50008/2013 - MM5G and and project PES3N (Ref: POCI-01-0145-FEDER-030629).

\section{REFERENCES}

1. Boccardi, F., R. Heath, A. Lozano, T. Marzetta, and P. Popovski, "Five disruptive technology directions for 5G," IEEE Communications Magazine, Vol. 52, No. 2, 74-80, Feb. 2014.

2. Marzetta, T., E. G. Larsson, H. Yangand, and H. Q. Ngo, Fundamentals of Massive MIMO, Cambridge University Press, 2016.

3. Bjornson, E., E. G. Larsson, and T. L. Marzetta, "Massive MIMO: Ten myths and one critical question," IEEE Communications Magazine, Vol. 54, No. 2, 114-123, 2016.

4. Astucia, V., P. Montezuma, R. Dinis, and M. Beko, "On the use of multiple grossly nonlinear amplifiers for higly efficient linear amplification of multilevel constellations," Proc. IEEE VTC2013-Fall, Las Vegas, NV, US, Sep. 2013.

5. Montezuma, P. and A. Gusmão, "Design of TC-OQAM schemes using a generalised nonlinear OQPSK-type format," IEE Elect. Letters, Vol. 35, No. 11, 860-861, May 1999.

6. Montezuma, P. and R. Dinis, "Implementing physical layer security using transmitters with constellation shaping," Proc. IEEE ICCCN2015, Las Vegas, NV, US, Aug. 2015.

7. Falconer, D., S. Ariyavisitakul, A. Benyamin-Seeyar, and B. Eidson, "Frequency domain equalization for single-carrier broadband wireless systems," IEEE Comm. Mag., Vol. 4, No. 4, 58-66, Apr. 2002.

8. Benvenuto, N., R. Dinis, D. Falconer, and S. Tomasin, "Single carrier modulation with nonlinear frequency domain equalization: An idea whose time has come again," Proc. IEEE 2010, Vol. 98, 69-96, 2010.

9. Ferreira, A., G. Gaspar, P. Montezuma, and R. Dinis, "Combining info and spatial directivities in multiple antenna transmission systems," IEEE YEF-ECE 2017, Costa da Caprica, May 2017.

10. Dinis, R., P. Montezuma, N. Souto, and J. Silva, "Iterative frequency-domain equalization for general constellations," IEEE Sarnoff Symposium, Princeton, USA, Apr. 2010.

11. Benvenuto, N. and S. Tomasin, "Block iterative DFE for single carrier modulation," IEE Elec. Let., Vol. 39, No. 19, 1144-1145, Sep. 2002.

12. Dinis, R., R. Kalbasi, D. Falconer, and A. Banihashemi, "Iterative layered space-time receivers for single-carrier transmission over severe time-dispersive channels," IEEE Comm. Letters, Vol. 8, No. 9, 579-581, Sep. 2004. 
13. Silva, F., R. Dinis, and P. Montezuma, "Estimation of the feedback reliability for IB-DFE receivers," ISRN Communications and Networking, Vol. 2011, Article No. 30, Jan. 2011.

14. Silva, P. and R. Dinis, Frequency-domain Multiuser Detection for CDMA Systems, River Publishers, Aalborg, 2012.

15. Borges, D., P. Montezuma, and R. Dinis, "Low complexity MRC and EGC based receivers for SC-FDE modulations with massive MIMO schemes," 2016 IEEE GlobalSip, Washington, DC, USA, Dec. 2016. 